Check for updates

Cite this: RSC Adv., 2018, 8, 30539

Received 16th July 2018

Accepted 22nd August 2018

DOI: $10.1039 / c 8 r a 06045 b$

rsc.li/rsc-advances

\section{Hypoglycemic, hypolipidemic and antioxidant effects of iridoid glycosides extracted from Corni fructus: possible involvement of the PI3K-Akt/PKB signaling pathway $\dagger$}

\author{
Jiefang Kang, $\xi^{\mathrm{a}}$ Chen Guo, (D) $+^{\mathrm{a}}$ Rodolfo Thome, ${ }^{\mathrm{b}}$ Ning Yang, ${ }^{\mathrm{a}}$ Yuan Zhang, ${ }^{\mathrm{a}}$ \\ Xing Li (D) *a and Xiaoyan Cao (D)*a
}

\begin{abstract}
Iridoid glycosides (CIG) are the major component of Corni fructus. In this work, we researched the antioxidative, hypoglycemic and lowering blood lipids effects of CIG on diabetic mice induced by a highfat diet (HFD) and streptozotocin (STZ). Furthermore, to investigate the molecular mechanism of action, the phosphorylation and protein expression of phosphoinositide 3-kinase (PI3K) and its downstream proteins, such as insulin receptor (INSR), protein kinase B (Akt/PKB) and glucose transporter 4 (GLUT4) have been detected. The results showed that CIG significantly improved oral glucose tolerance in diabetic mice. Biochemical indices also revealed that CIG had a positive effect on lipid metabolism and oxidative stress. In addition, CIG can significantly enhance the expression level of the PI3K-Akt/PKB pathway related proteins in skeletal muscle, which is the key pathway of insulin metabolism. These findings show that $\mathrm{CIG}$ can improve the hyperglycemia and hyperlipidemia of HFD-STZ-induced diabetic mice through the PI3K-Akt/PKB signaling pathway, and CIG might be a potential medicine or functional food for type 2 diabetes mellitus remedies.
\end{abstract}

\section{Introduction}

Diabetes is one of the most common chronic diseases in the world, and its prevalence is increasing dramatically. It was estimated that there were 425 million people with diabetes across the world in 2017, according to the International Diabetes Federation (IDF) programs, and this figure is expected to increase to 642 million by $2040 .{ }^{1}$ About $90 \%$ of these patients with diabetes have type 2 diabetes mellitus (T2DM). ${ }^{2}$ T2DM is a metabolic disease based on hyperglycemia characters and is due to insulin resistance (IR) caused by a progressive insulin secretory defect or by a lack of response of insulin receptors. ${ }^{3}$ Persistently high blood glucose levels may lead to a variety of long-term complications, which include but are not limited to cardiovascular and renal diseases, retinopathy, and poor blood flow. ${ }^{4}$ Although the existing management strategies for T2DM

\footnotetext{
${ }^{a}$ Key Laboratory of the Ministry of Education for Medicinal Resources and Natural Pharmaceutical Chemistry, National Engineering Laboratory for Resource Development of Endangered Crude Drugs in Northwest of China, College of Life Sciences, Shaanxi Normal University, Xi'an, Shaanxi, China. E-mail: caoxiaoyan@ snnu.edu.cn

${ }^{b}$ Department of Neurology, Thomas Jefferson University, Philadelphia, PA 19107, USA. E-mail: xingli_xian@126.com

$\dagger$ Electronic supplementary information (ESI) available. See DOI: $10.1039 / \mathrm{c} 8 \mathrm{ra06045b}$

\$ These authors contributed equally to this work.
}

have achieved great results, the tolerability and obvious side effects still cannot be ignored. ${ }^{5-7}$

Natural products with fewer side effects that are relatively inexpensive to use as novel therapies to treat diabetes are the focus of recent research. ${ }^{8,9}$ Corni fructus, the dried fruit of Cornus officinalis Sieb. et Zucc., also known as "Shan Zhu Yu" in Chinese, is an important crude herb used in Chinese medicine that possesses a variety of biological activities, such as antihyperglycemia, ${ }^{10}$ anti-inflammation, ${ }^{11}$ anti-oxidant, ${ }^{12}$ antiaging, ${ }^{13}$ and liver, neural and kidney protection. ${ }^{14,15}$ There are many chemical components in Corni fructus, including iridoid glycosides, flavonoids, organic acids, saccharides, and tannins. ${ }^{16}$ Iridoid glycosides are a group of active substance such as morroniside, loganin, cornuside and sweroside. Previously studies demonstrated that loganin had a protective effect against hepatic oxidative stress,${ }^{17}$ morroniside showed effective anti-inflammatory properties in AMI rats, ${ }^{18}$ sweroside could ameliorate liver injury by decreasing oxidative damage and inhibiting the production of proinflammatory kinases, ${ }^{19,20}$ and cornuside has been reported to possess immunomodulatory and anti-inflammatory activities. ${ }^{21,22}$ However, the underlying mechanisms of the therapeutic potential of CIG for diabetes have yet to be elucidated.

IR, a remarkable feature of T2DM, which was defined as a reduction in the physiological level of insulin by cells or tissues. is. ${ }^{23}$ Insulin signal transduction begins with the binding 
of insulin to insulin receptor (INSR) and involves the fine regulation of phosphorylation and dephosphorylation of signal molecules in intracellular signal transduction. ${ }^{\mathbf{2 4 2 5}}$ Tyrosine phosphorylation of insulin receptor substrate is induced through the combining of insulin and insulin receptor, followed by transduction of downstream enzymes, such as PI3K. ${ }^{26}$ PI3K is an intracellular phosphatidyl inositol kinase, with serine/threonine (Ser/Thr) kinase activity. A major effector of PI3K downstream signaling network is Akt, also named as PKB, a serine/threonine kinase. ${ }^{27}$ Studies have shown that PI3K-Akt/ PKB pathway could be induced by insulin and act as an indispensable effector. ${ }^{28}$ Akt/PKB can activate a large number of downstream molecules, such as translocating glucose transporters (GLUTs), especially by inducing GLUT4 to cell membrane to increase glucose consumption. ${ }^{29}$ Therefore, any defect in the Akt/PKB pathway and downstream molecules may lead to IR. ${ }^{30}$ Overall, activation of the PI3K-Akt/PKB pathway is necessary to promote peripheral glucose uptake and utilization.

Under this background, this study intended to probe the therapeutic role of CIG against HFD-STZ induced diabetic mice. Further explored is the underlying mechanism of anti-diabetic effects conveyed by CIG.

\section{Materials and methods}

\subsection{Chemicals and regents}

Crude CF were collected in Foping (Shaanxi, China), as areas in which the best quality of the herbs can be guaranteed. Raw materials were authenticated macroscopically and microscopically according to The Pharmacopoeia of the People's Republic of China (2015 Edition). Voucher specimens were deposited at the herbarium at College of Life Science, Shaanxi Normal University, China (the specimen number is CMQDA 201700011).

Streptozocin (STZ) was purchased from Sigma Chemical Co. (St Louis, MO, USA). Research Diets D12492 $60 \mathrm{kcal} \%$ fat were purchased from Yes Service Biotech (Shanghai, China). Morroniside, loganin, sweroside, and cornuside were obtained from Sigma Chemical Co. (St Louis, MO, USA). All antibodies were supplied by Cell Signaling Technology (Danvers, MA, USA), and all other chemicals and reagents were of the highest commercially available grade.

\subsection{Extraction of CIG}

Corni fructus powder was extracted with $80 \%$ methyl alcohol (1:20) for 9 min using an ultrasonic-microwave synergistic extraction system with a microwave power of $641 \mathrm{~W}$ and an ultrasonic power of $360 \mathrm{~W}$. The pooled extract was filtered and the solvent was removed by a vacuum rotary evaporator. Dissolved the concentrate in distilled water and purified using D101 macroporous resin elution with 50\% ethanol to obtain CIG. Quantification of CIG was performed by HPLC using a Shimadzu LC-2010A HPLC system (SHIMADZU, Kyoto, Japan). CIG was eluted with acetonitrile and $0.2 \%$ acetic acid, the flow rate was $1.0 \mathrm{~mL} \mathrm{~min}^{-1}$, the column temperature was $35^{\circ} \mathrm{C}$. The absorbance wavelength was set at $240 \mathrm{~nm}$ for quantification.

\subsection{Animals}

Seven-week-old male ICR mice (weight $18 \pm 2 \mathrm{~g}$ ) were obtained from the College of Medicine, Xi'an Jiaotong University (Xi'an, China). The mice were allowed to freely access to a standard laboratory rodent food and drinking water. All animals were maintained in a controlled environment at $25 \pm 1{ }^{\circ} \mathrm{C}$ and $60 \pm$ $5 \%$ relative humidity in a $12 \mathrm{~h}$ dark-light cycles and acclimatized for one week prior to use. All experiments using mice were performed in accordance with the National Institute of Health's Guide for the Care and Use of Laboratory Animals and were approved by the university's Institutional Animal Care and Use Committee of Shaanxi Normal University (Xi'an, China).

\subsection{Development of T2DM}

Before the experiments, all animals were accommodated to the laboratory environment for one week. T2DM mouse model was induced according to the previously published method with some modifications. ${ }^{31}$ Excluding the normal control (NC) group fed with normal diet (standard laboratory rodent food), other mice with a high fat diet (HFD) with $60 \% \mathrm{kcal} \%$ fat for 4 weeks. To induce diabetes, HFD-fed mice were fasted for $12 \mathrm{~h}$ before intraperitoneal injection of STZ solution (in citrate buffer, $\mathrm{pH}$ 4.2-4.5) at a dose of $40 \mathrm{mg}$ per $\mathrm{kg}$ body weight (bw), 5 times within one week; and the NC group received the citrate buffer alone in parallel. Seventy-two hours after the last injection, blood samples were removed from the tail vein of the over-night fasted mice. We elected mice with $\mathrm{FBG}>11.1 \mathrm{mmol} \mathrm{L}^{-1}$ for future study, which were recognized as T2DM.

\subsection{Experimental groups}

After the T2DM model was built successfully, the diabetic mice were randomized into 5 groups of 10 mice each. Grouping and treatment protocol was listed in Fig. 1. All mice were feeding for four weeks in a drinking and free drinking water. All mice weight and fasting blood glucose (FBG) were measured every week during the experiment. On the 29th day of the experiment, the mice were fasting for the night and collecting blood samples via the retro-orbital sinus of mice and separation of serum by centrifugation and were promptly placed in an environment of $-20{ }^{\circ} \mathrm{C}$ for later research. Following the mice were sacrificed via anaesthesia, tissues of pancreas, liver and muscle were carefully separated and washed with icy saline, and each part was fixed in $10 \%$ formalin ( $\mathrm{pH}$ 7.4) for biochemical and histological examinations.

\subsection{Oral glucose tolerance test (OGTT)}

OGTT was performed in overnight-fasted mice from all groups. Twenty minutes after sample administration (NC and DM groups were oral ingestion with normal saline), the mice were daily administered with glucose $2.0 \mathrm{~g} \mathrm{~kg}^{-1} \mathrm{bw}$. The samples of blood were harvested from the tail vein at 0 (before glucose administration), 30, 60, 120 and 180 min after glucose administration using One-Touch glucometer, which was purchased from HMD Bio Medical Co. (Taiwan, China). The result of OGTT 


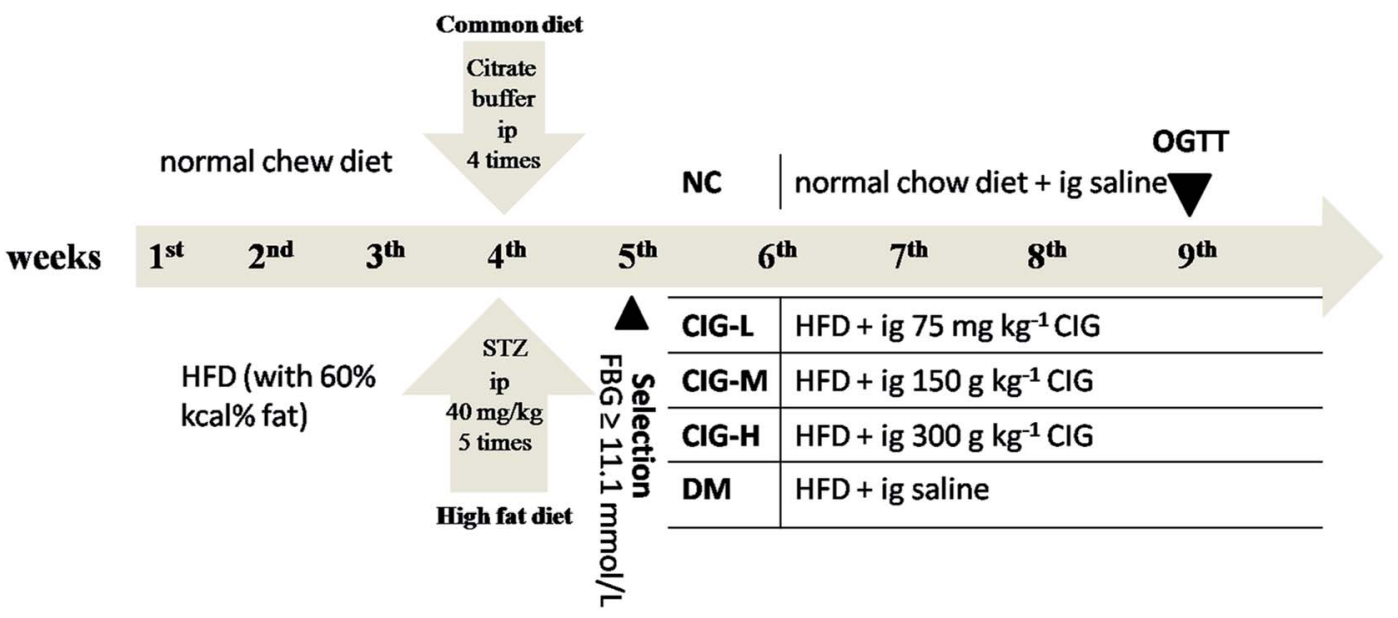

Fig. 1 Experimental design. ip, intraperitoneal injection; ig, intragastric administration.

was represented by an integrated area under the curve (AUC) calculated by GraphPad Prism version 6.0.

\subsection{Biochemical assay}

Serum total cholesterol (TC), serum triglycerides (TG), lowdensity lipoprotein cholesterol (LDL-C), high-density lipoprotein cholesterol (HDL-C), superoxide dismutase (SOD), and methylene dioxyamphetamine (MDA) were measured using the marketing diagnostic kit (Nanjing Jiancheng Bioengineering Institute, China). The serum fasting insulin level (FINS, as biochemical makers for serum insulin) and inflammatory cytokines (tumor necrosis factor (TNF)- $\alpha$, interleukin (IL)-6, Creactive protein (CRP)) were determined by ELISA assay kits (Tianjin Anoric Bio-technology CO., Ltd, Tianjin, China). All measurements were carried out according to the manufacturer's protocol of the corresponding kits. The homeostasis model assessment-insulin sensitivity index (HOMA-IS) and homeostasis model assessment-insulin resistance index (HOMA-IR) were calculated following the formulas HOMA-IS $=$ Ln $(1 /$ FINS $\times$ FBG $)$, HOMA-IR $=($ FBG $\times$ FINS $) / 22.5$, separately.

\subsection{H\&E staining}

Liver and pancreas from different groups were first fixed with $10 \%$ buffered formalin for $24 \mathrm{~h}$, and then buried in paraffin. Final sliced and stained with hematoxylin and eosin (H\&E). Histopathological changes of liver and pancreas were observed under a light microscope.

\subsection{Western-blotting}

After the mice were sacrificed, the protein concentration in muscle samples was determined by a BCA protein assay kit (Thermo Scientific, Waltham, MA, USA). And western blot were analyzed according to the previously method with some modification..$^{32}$ Briefly, following the tissue was lysed by (radio immunoprecipitation assay) RIPA buffer on ice, the protein samples were then separated on a $10 \%$ sodium dodecyl sulfate polyacrylamide gel electrophoresis (SDS-PAGE), transferred to nitrocellulose membrane (Millipore, MA, USA), and blotted with primary antibodies at $4{ }^{\circ} \mathrm{C}$ overnight. The following primary antibodies were used: INSR, p-INSR, PI3K, p-PI3K, Akt/PKB, pAkt/PKB, and GLUT4. The membranes were shaken in a solution of secondary antibody (peroxidase-conjugated affinipure goat anti-rabbit or anti-mouse $\mathrm{IgG}$ ) for $1 \mathrm{~h}$. Infrared fluorescence were conducted on an Odyssey infrared imaging system (Li-cor Bioscience, Lincoln, NE, USA). Anti- $\beta$-actin was used to ensure equal loading. Quantification was performed using the computerized imaging program, Quantity One (Bio-Rad).

\subsection{Statistical analysis}

Statistical analysis was performed using GraphPad.Prism.v.5.0. All determinations were done at least in triplicate and all were averaged. Values were expressed as means \pm standard deviations (SD). Analysis of variance (ANOVA) was employed to determine the statistical significance. And a value of $p<0.05$ was considered to be statistically significant.

\section{Results}

\subsection{HPLC analysis}

As shown in Fig. 2 and Table 1, CIG was purified by a multistep purification procedure including EtOH extraction and D-101 macroporous adsorption resin purification. The contents of morroniside, loganin, sweroside, and cornuside at concentrations of $1.523 \%, 0.696 \%, 0.059 \%$, and $0.169 \%$ of the dried fruit, respectively. The employed HPLC method was validated in performing the quantitative analysis (Table 2). These results for method validation suggested that the HPLC method established in our study was reliable for evaluating CIG of Corni fructus.

\subsection{Effect of CIG on body weight and organ index}

In this study, the concentration of selected CIG extract dose (75, $150,300 \mathrm{mg} \mathrm{kg}{ }^{-1}$ ) were basing on previous studies, ${ }^{33}$ where the extracts researched have similar composition as CIG and show a hypoglycemic effect. The body weight of each group was shown in Table 3. The mice in DM group was significantly lighter than that of the NC group during the experiment period $(p<0.05)$. Diabetic mice that received 4 weeks of CIG were 
(A)

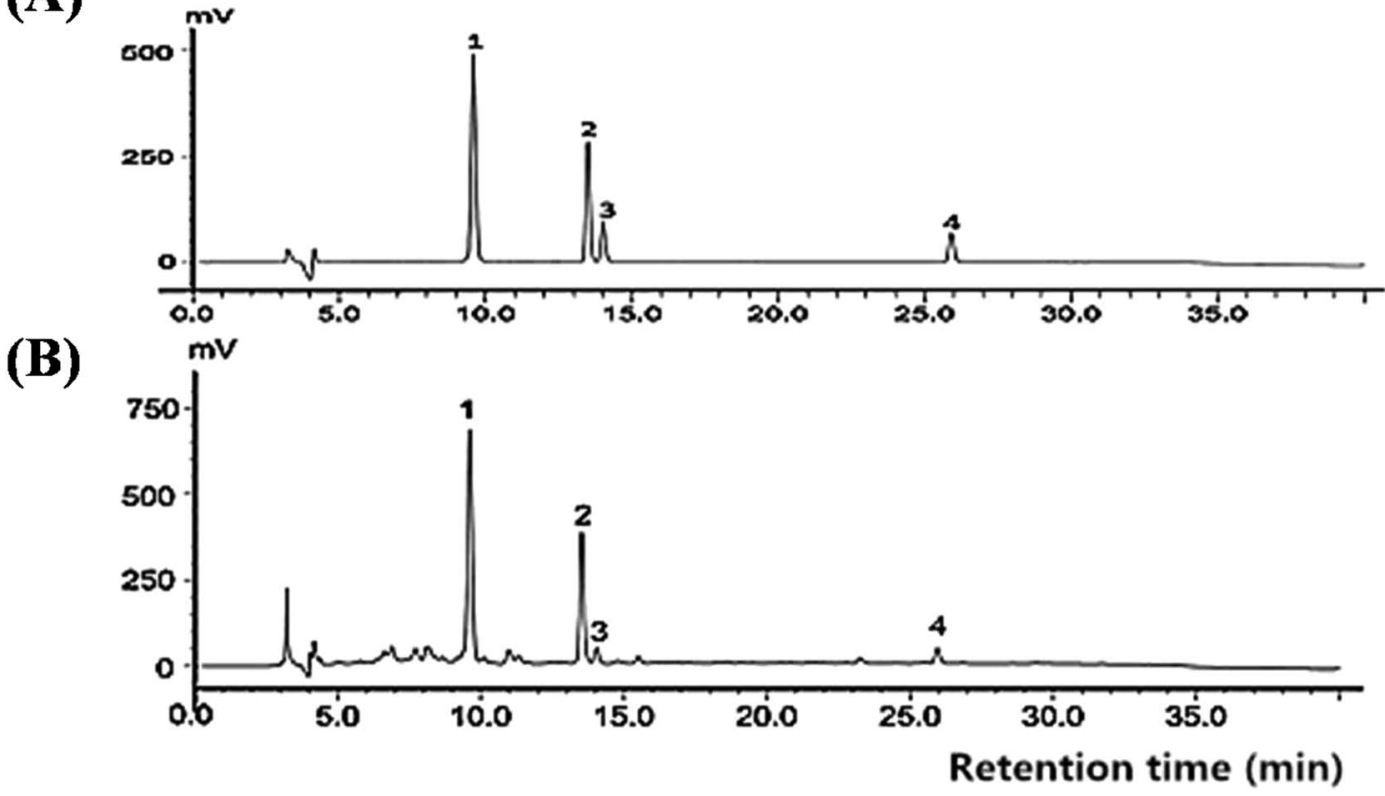

Fig. 2 HPLC chromatogram of (A) reference substances and (B) iridoid glycosides from Corni fructus. Peaks: 1, morroniside; 2, loganin; 3, sweroside; 4, cornuside.

Table 1 The composition of morroniside, loganin, sweroside and cornuside in $\mathrm{CIG}$

\begin{tabular}{llll}
\hline Peak no. & Assigned identity & $\begin{array}{l}\text { Retention time } \\
(\mathrm{min})\end{array}$ & $\begin{array}{l}\text { Contents in } \\
\text { CIG }\left(\mathrm{mg} \mathrm{g}^{-1}\right)\end{array}$ \\
\hline 1 & Morroniside & 9.71 & 76.63 \\
2 & Loganin & 13.62 & 25.26 \\
3 & Sweroside & 14.16 & 2.98 \\
4 & Cornuside & 26.14 & 8.45 \\
\hline
\end{tabular}

significantly increased in body weight compared to that of DM group $(p<0.05)$. After CIG gavage, the weight of the mice in this group began to increase, which could be due to the increase of unused fat-intake; however, these weights started to decrease after second week, which might be the result of diabetes severity.

The liver, kidney and spleen index of group DM was significantly higher than that of group NC $(p<0.05)$. However, after administration of CIG for 4 weeks, CIG restraining these trends and the related tissue index returned to normal level (Fig. 3).

\subsection{FBG, OGTT and FINS levels}

Abnormal glycemic change is a typical characteristic of diabetes. As shown in Fig. 4, there was a notable increase in FBG of DM group when compared to NC group. Compared with the DM group, the FBG levels in CIG-treated group decreased significantly after 4 weeks. Accordingly, OGTT showed that the blood glucose reached its peak within $30 \mathrm{~min}$ and gradually decreased in the NC group. The CIG-H group exhibited a lower FBG than DM group at $30 \mathrm{~min}$, which indicates that CIG effective inhibition of the peak of glucose. A lower AUC indicates the higher efficiency of OGTT and our data show that all the CIG groups had a remarkable low-level of AUC values compared to DM control. These results indicate that CIG have a positive effect on blood glucose regulation.

FINS level of the mice in DM group was abnormally lower than the NC group $(p<0.05)$ (Table 4$)$, which shown that the islets were destroyed. However, this trend has been markedly suppressed by CIG and the FINS level has been raised in CIGtreated groups (CIG-L increased by $11.11 \%$, CIG-M increased by $29.20 \%$, CIG-H increased by $50.85 \%, p<0.05$ ). In addition, the study investigated whether or not CIG could exert an

Table 2 Method validation of morroniside, loganin, sweroside and cornuside by HPLC analysis ${ }^{a}$

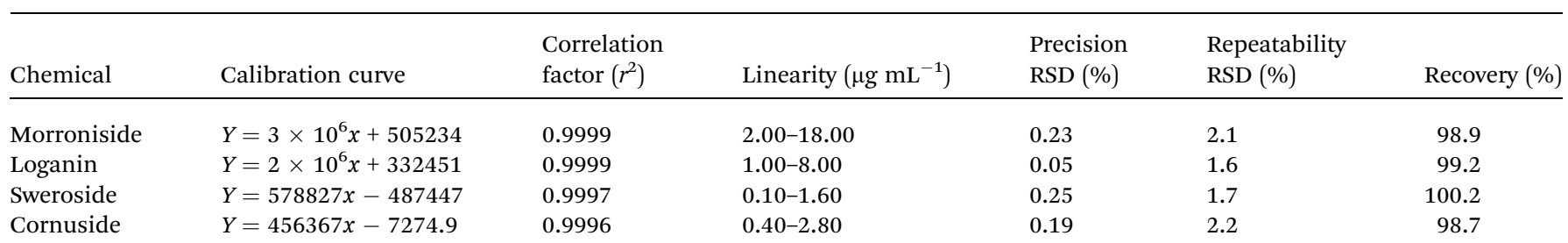

${ }^{a}$ These samples were subjected to HPLC analysis. The calibration curve was used to calibrate the concentration of various chemical constituents. The mean values were expressed here, and the standard error of the mean (SEM) values of the six tested chemicals were less than $5 \%$ of the mean. The calibration was repeated 3 times $(n=3)$. 
Table 3 Effects of CIG on body weight of experimental mice ${ }^{a}$

\begin{tabular}{|c|c|c|c|c|c|}
\hline Group & \multicolumn{5}{|c|}{ Body weight (g) } \\
\hline CIG-L & $30.50 \pm 1.93$ & $30.77 \pm 0.63$ & $30.75 \pm 1.31$ & $30.6 \pm 0.78$ & $30.45 \pm 0.54$ \\
\hline CIG-M & $31.45 \pm 0.88$ & $31.80 \pm 1.48$ & $30.0 \pm 1.62^{*}$ & $30.5 \pm 1.95$ & $30.19 \pm 2.27$ \\
\hline CIG-H & $31.33 \pm 1.54$ & $31.53 \pm 1.36$ & $30.78 \pm 2.24^{*}$ & $31.2 \pm 2.66^{\#}$ & $31.12 \pm 2.66^{\#}$ \\
\hline
\end{tabular}

${ }^{a}$ Values are expressed as means \pm SD of 10 mice in each group, where $p<0.05$ denotes statistically significant difference with respect to. * NC group; \#, DM group.

insulin sensitivity and resisting activity in T2DM mice. As shown in Table 4, in the presence of CIG, the diabetic mice showed a tendency of enhanced HOMA-IS and HOMA-IR. These results indicated that CIG ameliorates IR in diabetic mice.

\subsection{Effects of CIG on serum lipid profiles of diabetic mice}

Diabetes patients often have disorders in the lipid metabolism. To test whether CIG would have a protective effect on lipid metabolism, the effects of CIG on biochemical indicators of blood lipid in experimental mice are summarized in Fig. 5. TC, TG and LDL-C levels of DM were significantly enhanced compared with that of NC. Such levels decreased after the administration of CIG. On the other hand, while HDL-C level of DM remarkably declined compared with that of NC, such level of those administered with CIG increased. These results show that administration of CIG could ameliorates the lipid profiles of diabetic mice especially at the dose of 150 and $300 \mathrm{mg} \mathrm{kg}^{-1}$.

\subsection{The effects of CIG on inflammatory cytokines}

As abnormalities in inflammation is another characteristic of diabetes, we also analyzed the levels of the inflammatory cytokines, including IL-6, TNF- $\alpha$, and CPR in the liver of experimental mice. Our results showed that under the treatment of CIG at $150 \mathrm{mg} \mathrm{kg}^{-1}$ (CIG-M) and $300 \mathrm{mg} \mathrm{kg}^{-1}$ (CIG-H), the expression of IL-6, TNF- $\alpha$ and CRP were significantly reduced compared with DM control (Fig. 6). These data indicates that CIG has the ability to improve the inflammatory damage of the liver.

\subsection{Effects of CIG on SOD and MDA level in liver}

MDA is one of the important products of lipid peroxidation, and SOD is an important antioxidant enzyme in organism. The content of MDA and SOD are common used to reflect the degree of lipid peroxidation. As shown in Fig. 7, the level of MDA in DM group was markedly enhanced compared with NC group. After treat with CIG, the activity of MDA in CIG-M and CIG-H was obviously lower than that in DM $(p<0.05)$. Opposite results were observed regarding in the activities of SOD, which plays a pivotal role in the oxidation and antioxidant balance. However, there was no significant difference in the SOD and MDA activity of the CIG-L when compared with that in group DM.

\subsection{CIG improved histopathological damage in the liver and pancreas}

Histopathological analyses revealed that STZ induces severe injury to the liver and pancreas (Fig. 8). Liver from mice of the NC group displayed a complete and clear structure, normal and regular hepatocytes, and uniform cytoplasm. By contrast, DM
(A)

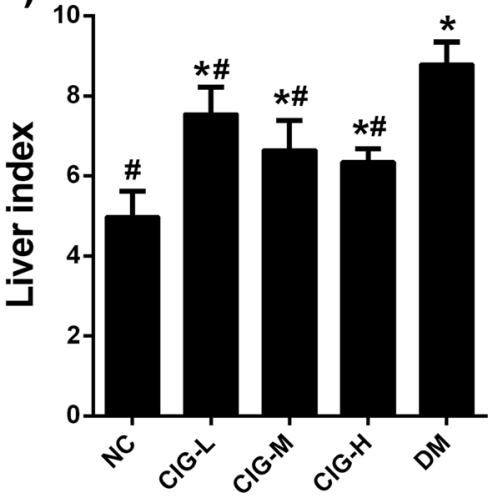

(B)

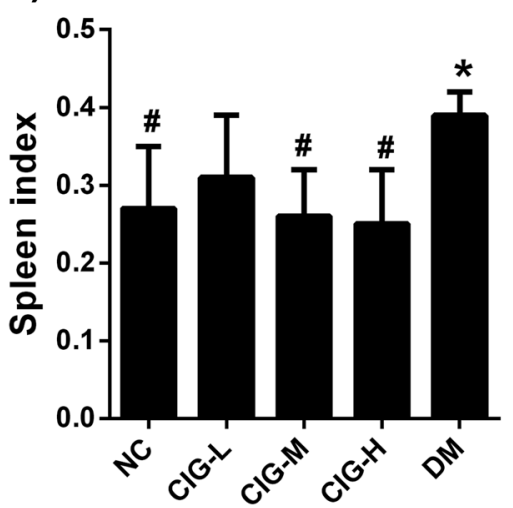

(C)

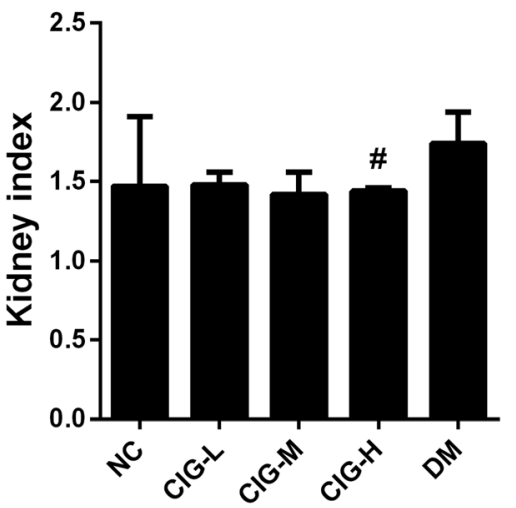

Fig. 3 Effects of CIG on organ weight index of experimental mice. (A) Liver index; (B) spleen index; (C) kidney index; NC, normal control group; CIG-L, diabetes mice treated with $75 \mathrm{mg} \mathrm{kg}^{-1} \mathrm{CIG}$ per day; CIG-M, diabetes mice treated with $150 \mathrm{mg} \mathrm{kg}^{-1} \mathrm{CIG}$ per day; CIG-H, diabetes mice treated with $300 \mathrm{mg} \mathrm{kg}^{-1} \mathrm{CIG}$ per day; DM, diabetic mice treated with drinking water. We evaluated $p<0.05$ denotes statistically significant difference with respect to. *, NC group; \#, DM group. 
(A)
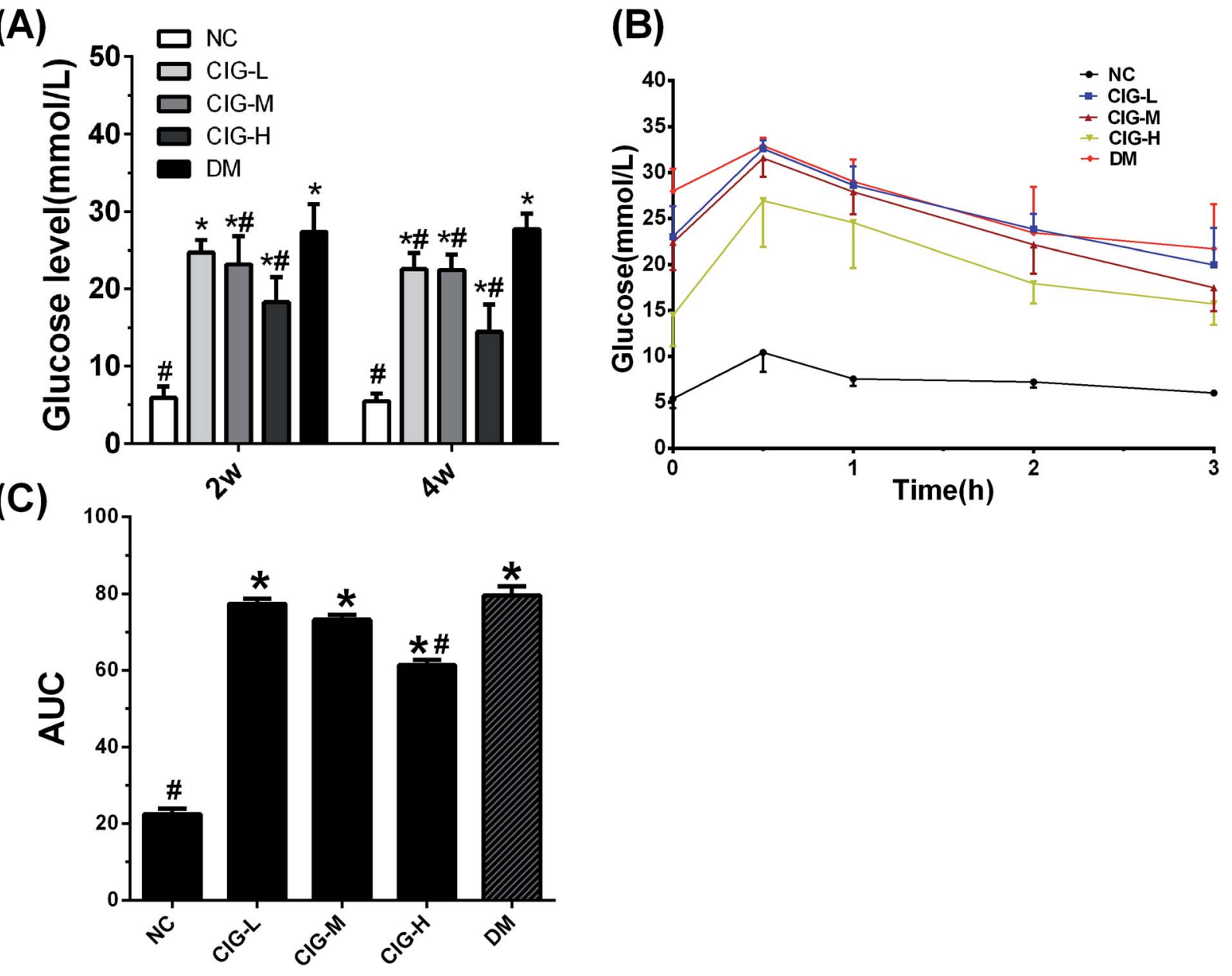

Fig. 4 Effects of CIG on glycemic modulation of experimental mice. (A) FBG; (B) OGTT; (C) AUC; NC, normal control group; CIG-L, diabetes mice treated with $75 \mathrm{mg} \mathrm{kg}^{-1} \mathrm{CIG}$ per day; $\mathrm{ClG}-\mathrm{M}$, diabetes mice treated with $150 \mathrm{mg} \mathrm{kg}^{-1} \mathrm{ClG}$ per day; $\mathrm{ClG}-\mathrm{H}$, diabetes mice treated with $300 \mathrm{mg} \mathrm{kg}^{-1} \mathrm{CIG}$ per day; DM, diabetic mice treated with drinking water. We evaluated $p<0.05$ denotes statistically significant difference with respect to. *, NC group; \#, DM group.

Table 4 Effects of CIG on fasting insulin, insulin sensitivity index and insulin resistance index of experimental mice ${ }^{a}$

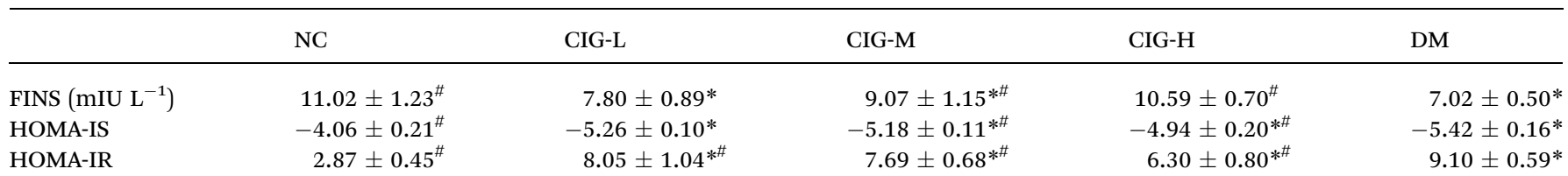

${ }^{a}$ Data are expressed as the means \pm standard deviation (SD) for 10 mice in each group, where $p<0.05$ denotes statistically significant difference with respect to, *, NC group, \#, DM group. HOMA-IR $=($ FBG $\times$ FINS $) / 22.5$. HOMA-IS $=$ Ln $(1 /$ FINS $\times$ FBG $)$.

mice displayed severe damage with a disorder of the hepatocytes and the cytoplasm appeared as a vacuole. The CIG treatments significantly prevented the damage to the liver (Fig. 8A)

It had been generally believed that IR is associated with insufficient insulin secretion that can induce the damages on pancreatic $\beta$-cells. ${ }^{34}$ The results of the pancreatic pathology are shown in Fig. 8B. Group NC had complete pancreatic structure and a large number of neatly arranged islet beta cells. In opposite, pancreatic cells in DM group were injured obviously, focal necrosis and with sparse. However, CIG-treated groups were alleviating the injury in different degrees. All the histopathological results showed that CIG could delay and treat the damage of liver and pancreas, and have protective effects on HFD-STZ induced diabetic mice. Therefore, CIG could be a potential resource for the prevention and treatment of diabetes.

\subsection{CIG promoted INSR, PI3K, Akt/PKB, and GLUT4 phosphorylation in skeletal muscle of diabetic mice}

As one of the major pathogenic mechanism of diabetes, IR results from a variety of factors. ${ }^{35}$ IR occurs when binding of insulin and insulin receptor is compromised or when signal 
(A)

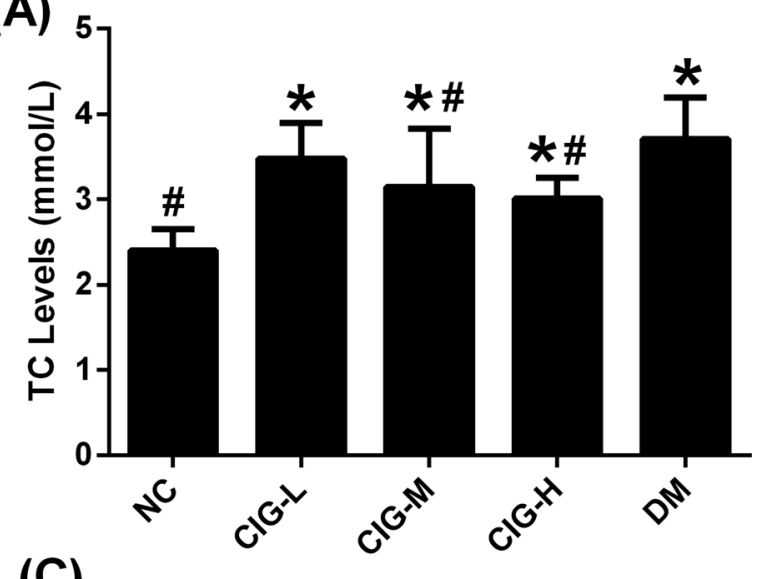

(C)

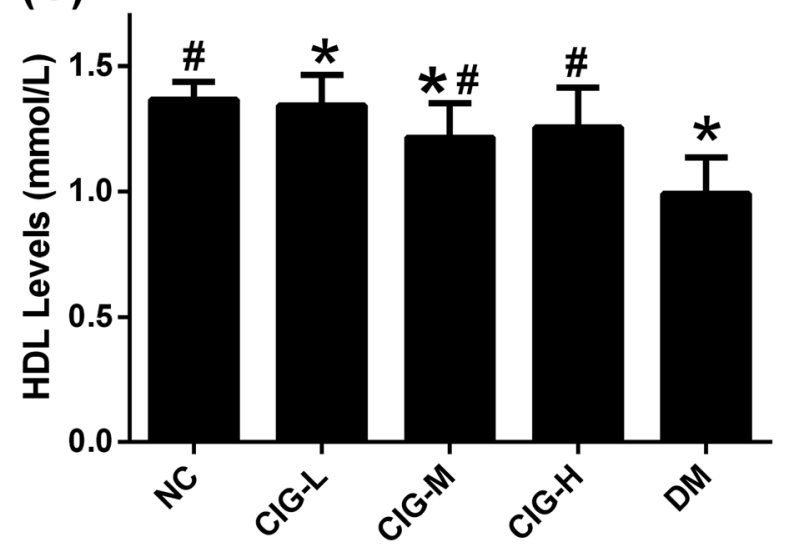

(B)
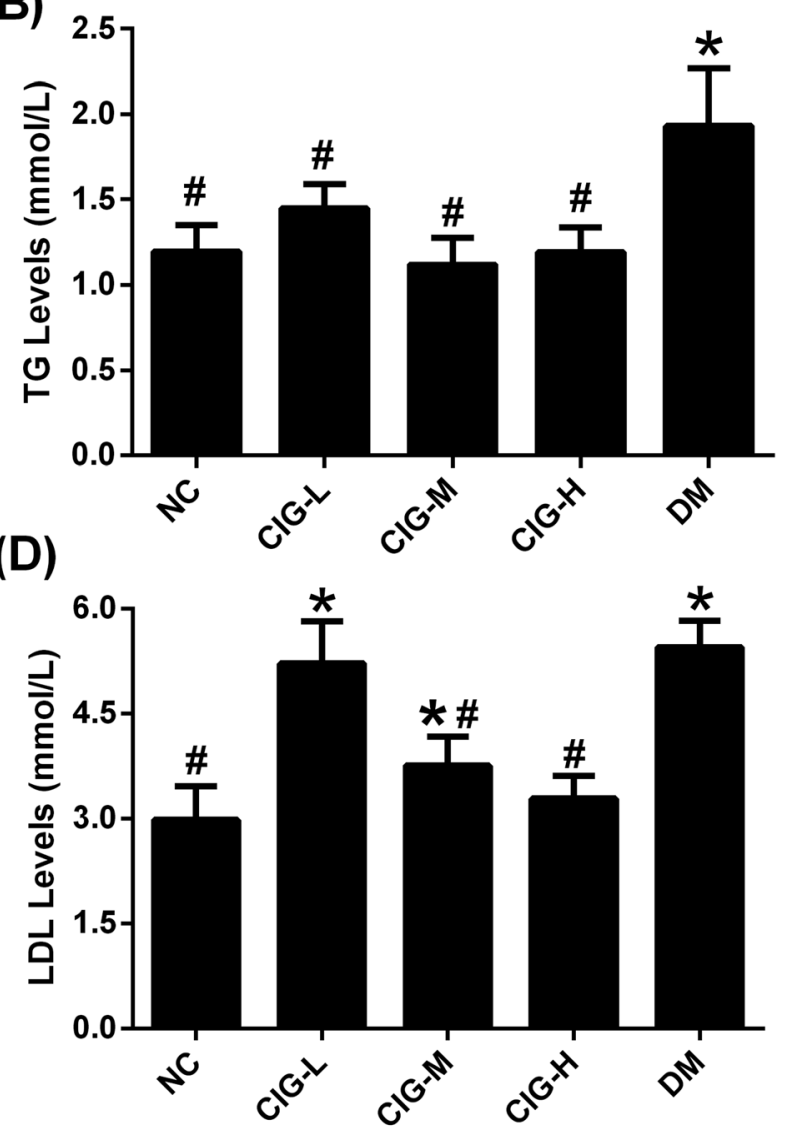

Fig. 5 The effects of CIG on the serum lipid profiles of experimental mice. (A) TC; (B) TG; (C) HDL; (D) LDL; NC, normal control group; CIG-L, diabetes mice treated with $75 \mathrm{mg} \mathrm{kg}^{-1} \mathrm{CIG}$ per day; $\mathrm{CIG}-\mathrm{M}$, diabetes mice treated with $150 \mathrm{mg} \mathrm{kg}^{-1} \mathrm{CIG}$ per day; CIG-H, diabetes mice treated with $300 \mathrm{mg} \mathrm{kg}^{-1} \mathrm{CIG}$ per day; DM, diabetic mice treated with drinking water. We evaluated $p<0.05$ denotes statistically significant difference with respect to. *, NC group; \#, DM group.

transduction from the insulin receptor is not completed. ${ }^{36}$ triggered by insulin. To study the mechanistic effect of CIG on INSR, PI3K, Akt/PKB and GLUT4 are key molecules involved in IR, western blot analysis of INSR, PI3K, Akt/PKB, and GLUT4 glucose uptake and in the signal transduction pathway protein levels in skeletal muscle were carried out. Our data

(A)

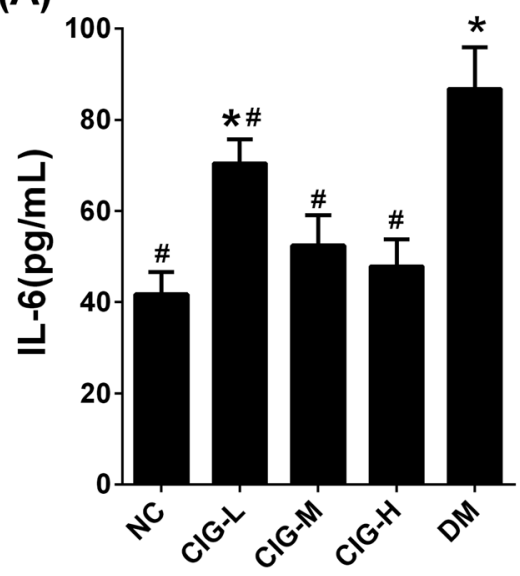

(B)

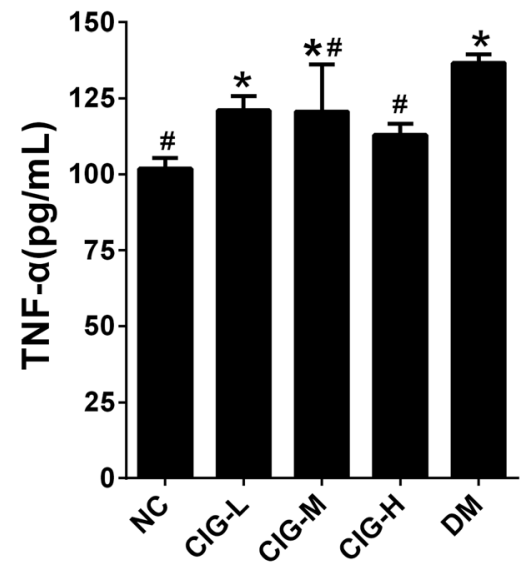

(C)

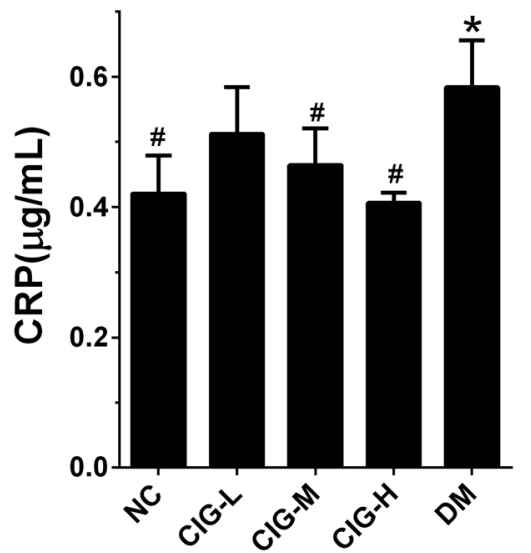

Fig. 6 The effects of CIG on liver inflammatory cytokines of experimental mice. (A) II-6; (B) TNF- $\alpha$; (C) CRP. NC, normal control group; CIG-L, diabetes mice treated with $75 \mathrm{mg} \mathrm{kg}^{-1} \mathrm{CIG}$ per day; $\mathrm{CIG}-\mathrm{M}$, diabetes mice treated with $150 \mathrm{mg} \mathrm{kg}^{-1} \mathrm{CIG}$ per day; CIG-H, diabetes mice treated with $300 \mathrm{mg} \mathrm{kg}^{-1} \mathrm{CIG}$ per day; DM, diabetic mice treated with drinking water. We evaluated $p<0.05$ denotes statistically significant difference with respect to. *, NC group; \#, DM group. 
(A)

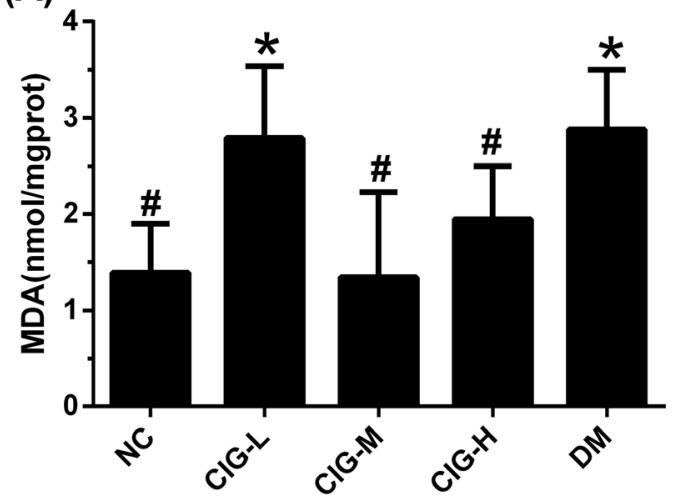

(B)

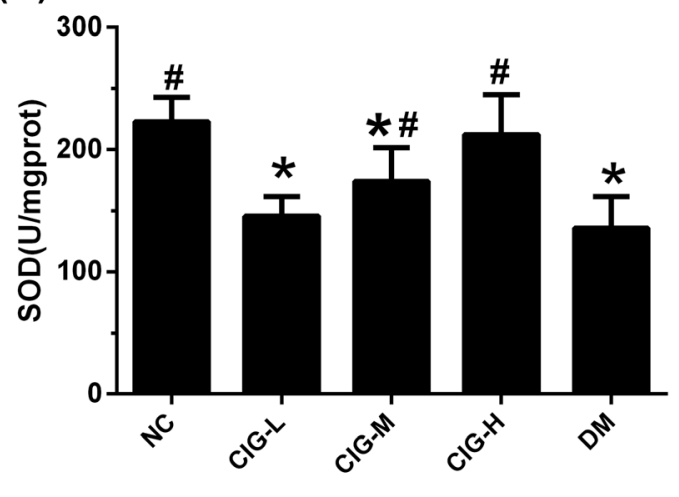

Fig. 7 The effects of CIG on liver oxidative stress related factors of experimental mice. (A) MDA; (B) SOD; NC, normal control group; CIG-L, diabetes mice treated with $75 \mathrm{mg} \mathrm{kg}^{-1} \mathrm{CIG}$ per day; $\mathrm{CIG}-\mathrm{M}$, diabetes mice treated with $150 \mathrm{mg} \mathrm{kg}^{-1} \mathrm{ClG}$ per day; CIG-H, diabetes mice treated with $300 \mathrm{mg} \mathrm{kg}^{-1} \mathrm{CIG}$ per day; DM, diabetic mice treated with drinking water. We evaluated $p<0.05$ denotes statistically significant difference with respect to. *, NC group; \#, DM group.

showed that the phosphorylation of these four proteins in skeletal muscle was significantly decreased in DM compared with the group of NC, while CIG-H restored the expression of INSR, PI3K, Akt/PKB, and GLUT4 (Fig. 9).

\section{Discussion}

Earlier studies have shown Corni fructus that exhibits immune regulating function, anti-shock effects and protective effect on experimental diabetic nephropathy. ${ }^{37,38}$ Another work has also raised the possibility that iridoid glycosides might achieve anti-diabetic effect by inhibiting the phosphorylase of glycogen ${ }^{39}$ in particular, morroniside was suggested to play an important role in preventing and/or postponing the occurrence of diabetic kidney disease. According to the previous studies, the contents of morroniside, sweroside, loganin and cornuside in Corni fructus were very rich. ${ }^{40}$ Thus, we could assume that CIG not only possesses effects similar to those of iridoid glycosides, but many also have other functions beyond the extracted CIGs. In order to develop and utilize of CIG to the extent of industrial level, the effects of CIG on diabetes were studied.
(A)
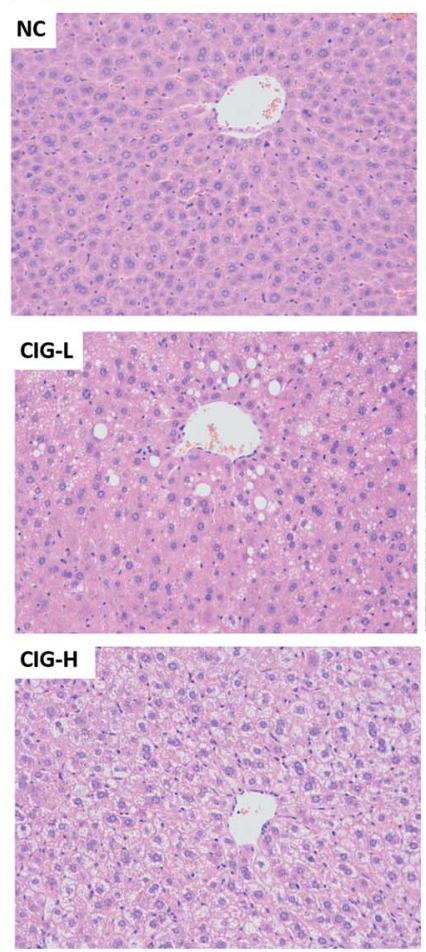

(B)
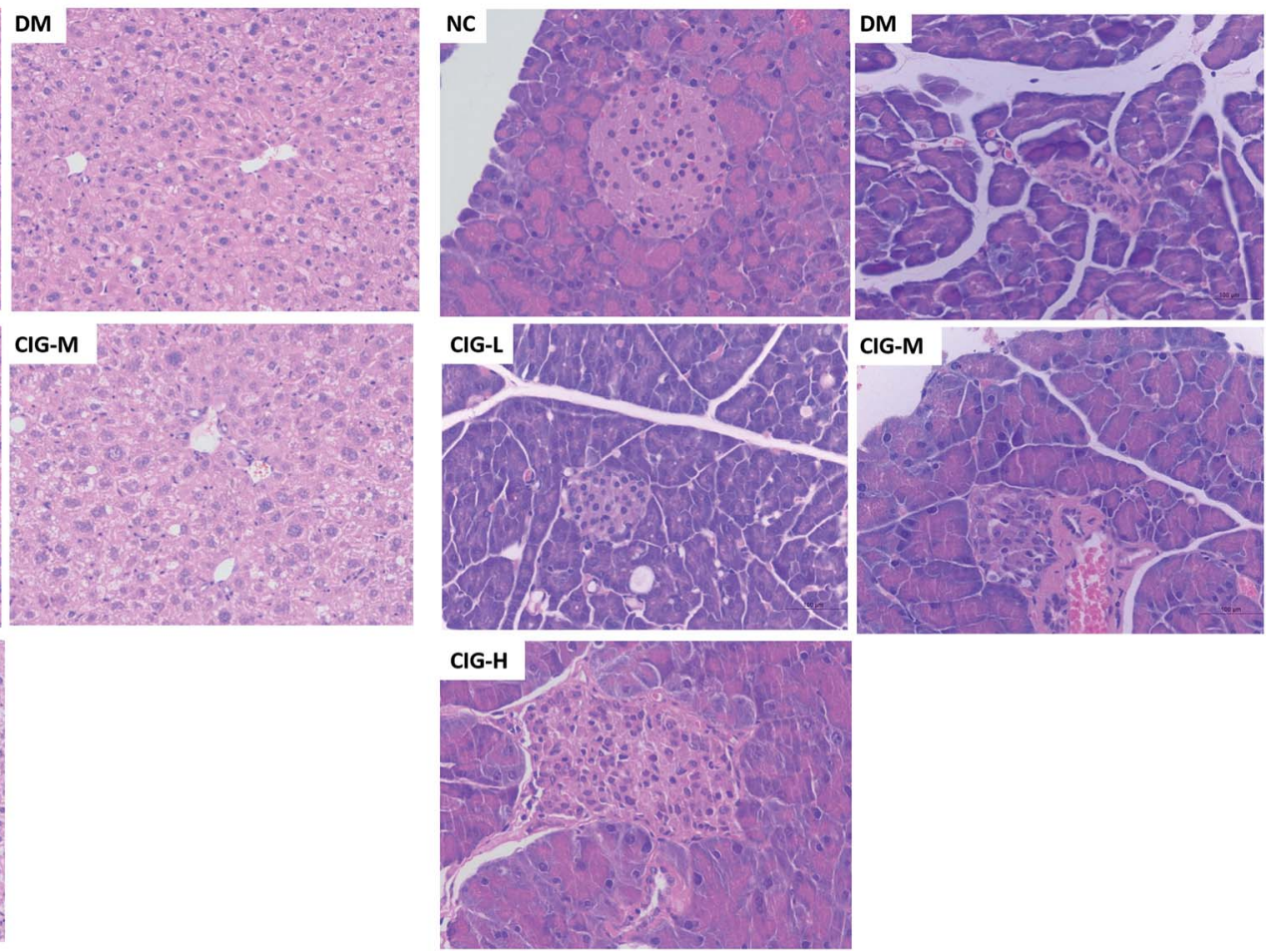

Fig. 8 Histopathological changes in the liver (A) (HE staining 200x) and pancreas (B) (HE staining 400x). 
(A)

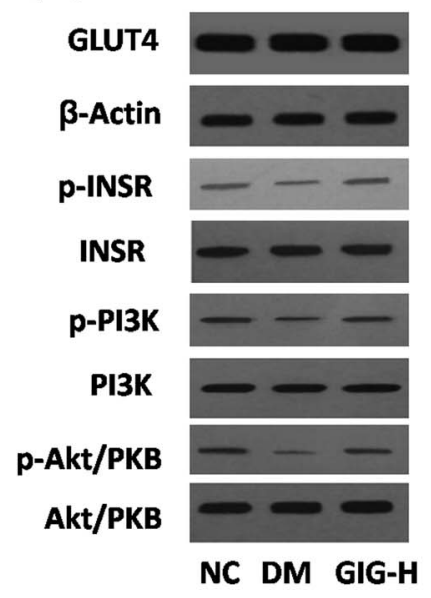

(B)

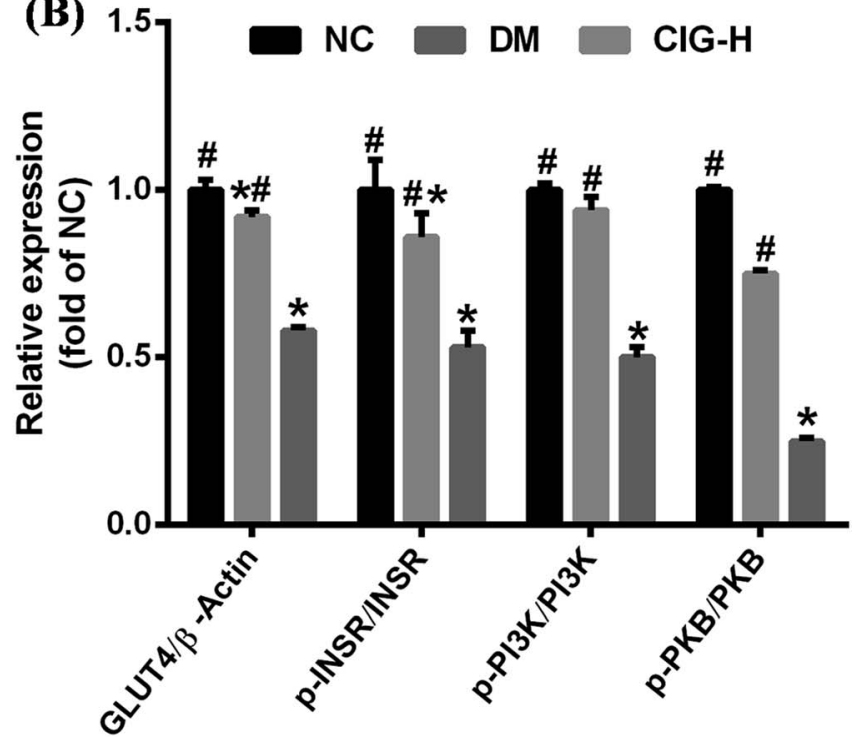

Fig. 9 Protein status and gene expressions of key enzymes of glucose metabolism in skeletal muscle. NC, normal control group; CIG-H, diabetes mice treated with $300 \mathrm{mg} \mathrm{kg}^{-1} \mathrm{CIG}$ per day; DM, diabetic mice treated with boiled water. We evaluated $p<0.05$ denotes statistically significant difference with respect to. *, NC group; \#, DM group.

In this paper we show that CIG reduces the severity of HFDSTZ induced diabetes through the modulation of the insulin signaling pathway. First of all, ICR mice became diabetes mellitus induce by HFD and multiple low-dose STZ injections. ${ }^{41}$ After the diabetes model was established, CIG gavage was used at the doses of 75 (low dose), 150 (medium dose) and 300 (high dose) $\mathrm{mg}$ per $\mathrm{kg}$ per day in the experiment. HFD/STZ-induced diabetic mice administrated with CIG daily were investigated for the effects on various parameters, including body weight, FBG, lipid level, FINS, antioxidant ability, inflammatory cytokines and other parameters involving liver, pancreas and skeletal muscle. As shown in Table 3, while body weights of the CIGtreated HFD/STZ-induced diabetic mice increases, the increase of FBG levels was significantly suppressed (Fig. 4A). This result suggested that CIG may delay dietary carbohydrates' absorption, resulting the blood glucose level decrease.

Because impaired OGTT is an important criterion for diabetes. The OGTT was thus conducted and the results were shown in Fig. 4B. Reports have previously demonstrated the beneficial action of Corni fructus on diet-induced IR and glucose intolerance..$^{42}$ In this research, OGTT indicated that the AUC of glucose tolerance in CIG was lower appreciably than that in the DM $(p<0.05)$ (Fig. 4B and C), showing that CIG has an effective improvement of glucose tolerance in diabetic mice. IR is the main symptom of T2DM, which is related to the injury of insulin secretion and hyperglycemia caused by STZ injection. In this research, the insulin levels in group CIG were significantly higher than those in group DM, suggesting that CIG stimulates insulin secretion in islets of diabetic mice. IR was defined as HOMA-IR $>2.6,{ }^{43-45}$ a higher level of HOMA-IR and lower HOMAIS showed the dysfunction of $\beta$-cell in DM group mice (Table 4). However, the HOMA-IR and HOMA-IS levels of all CIG mice were significantly reduced and increased, demonstrating that CIG could ameliorate $\beta$-cell function and IR.
Moreover, cinnamon polyphenols longstanding IR will also interfere with the metabolism of glucose and lipid, leading to a further rise in glycolipid levels. ${ }^{46}$ Hyperlipidemia and dyslipidemia are closely related to diabetes, which is a metabolic disorder caused by diabetes. ${ }^{47}$ In this research, CIG intake obviously depressed TC, TG levels and increased HDL levels in the mice of DM group (Fig. 5). Elevated levels of TC and TG are associated with high diabetes risk, while increased HDL levels can alleviate diabetes. ${ }^{46}$

As we all know, oxidative stress plays a key role in the pathogenesis of diabetes and his complications. ${ }^{48,49}$ And some cytokines like IL-6, TNF- $\alpha$ and CRP, also have been related to the risk of developing T2DM and cardiovascular diseases that are strongly associated with IR and body fat amount. ${ }^{50}$ Oxidative stress and inflammation can interact, IL- 6 and TNF- $\alpha$ can stimulate the production of reactive oxygen species, which could activate a variety of signal transduction mechanisms in the cell, and leads to IR eventually. ${ }^{51}$ Consistently, DM mice herein had markedly enhanced lipid peroxidation product MDA, and reduced activities of antioxidant enzymes SOD. ${ }^{52,53}$ In the present study, we found that CIG could inhibit the expression of IL-6, TNF- $\alpha$, CRP and MDA, and increase the SOD levels in the liver. These results suggest that CIG blocks the pathogenesis of diabetes by inhibiting the production of proinflammatory cytokines.

Aside from the IR of liver, the IR of muscle and adipose are the two other main affected organs in diabetes. Skeletal muscle plays an important role in glucose and lipid homeostasis via regulation of the insulin signaling pathway. ${ }^{54}$ To clarify the mechanism of CIG in treating diabetes, we detected the mRNA and protein expression of the main targets of the insulin transduction pathway in skeletal muscle. Insulin exerts its action through the activation of PI3K by the INSR. INSR is activated by tyrosine phosphorylation and is the central 
mediator in the insulin response upstream of PI3K. ${ }^{55}$ Many studies have shown that Akt/PKB plays an important role in insulin action, including increased glucose uptake and GLUT4 translocation. ${ }^{56}$ Therefore, increase of Akt/PKB activity was considered to be closely connected to the increase of glucose uptakes and insulin signaling cascade activation, and Akt/PKB is the primary target of PI3K-generated signals. ${ }^{28}$ Akt/PKB activates AS160 (Akt substrate, $160 \mathrm{kDa}$ ), which promotes the translocation of GLUT4 and absorption of glucose in muscle cells. More recently, evidence showed that increasing the secretion of GLUT4 in adipose or skeletal muscles reverse $\beta$-cell from necrosis. ${ }^{57}$ Polysaccharides from Ganoderma lucidum were also shown to be involved in the modulation of GLUT4 expression in the treatment of diabetic mice. ${ }^{58}$ Therefore, PI3K-Akt/ PKB signaling pathway plays a key part in metabolic effect. ${ }^{59}$ The lower expression of PI3K, Akt/PKB and GLUT4 expression levels in STZ-HFD induced diabetic mice compared to control mice suggested that glucose uptake via GLUT4 into cells through PI3K-Akt/PKB signal pathway was inhibited. In our findings, CIG could increase the protein expression of INSR, PI3K, Akt/PKB, and GLUT4 in skeletal muscle, demonstrating that the hypoglycemic activity of CIG is mediated by the PI3KAkt/PKB signaling pathway, which could improve IR.

\section{Conclusion}

In conclusion, we demonstrate that CIG has a hypoglycemic, hypolipidemic and antioxidant effects in diabetes. The possible mechanism of this effect may be related to the activation of the PI3K-Akt/PKB signaling pathway. CIG could be developed as a potential therapeutic treatment for diabetes.

\section{Conflicts of interest}

The authors declare no competing financial interest.

\section{Acknowledgements}

This work received supported from National Key Technologies R \& D Program for Modernization of Traditional Chinese Medicine (Grant No. 2017YFC1701300) and Major Project of Shaanxi Province, China (Grant No. 2017ZDXM-SF-005).

\section{References}

1 I. D. Federation, IDF Diabetes Atlas,http://www.idf.org/ diabetesatlas.

2 A. D. Association, Diabetes Care, 2017, vol. 40, p. S11.

3 A. D. Association, Diabetes care 2014, 2014, vol. 37, pp. S14S80.

4 J. L. Ríos, F. Francini and G. R. Schinella, Planta Med., 2015, 81, 975.

5 W. Ma, K. J. Wang, C. S. Cheng, G. Q. Yan, W. L. Lu, J. F. Ge, Y. X. Cheng and N. Li, J. Ethnopharmacol., 2014, 153, 840.

6 K. W. Ong, A. Hsu and B. K. H. Tan, Biochem. Pharmacol., 2013, 85, 1341.
7 G. A. Nichols, T. M. Kimes, J. B. Harp, T. D. Kou and K. G. Brodovicz, Diabetes Care, 2012, 35, 495-497.

8 W. Tang, S. Li, Y. Liu, M. T. Huang and C. T. Ho, J. Funct. Foods, 2013, 5, 1784-1793.

9 F. F. Anhê, Y. Desjardins, G. Pilon, S. Dudonné, M. I. Genovese, F. M. Lajolo and A. Marette, PharmaNutrition, 2013, 1, 105-114.

10 H. J. Kim and Y. C. Kim, Mol. Cell. Toxicol., 2010, 6, 135-142. 11 Y. H. Sung, H. K. Chang, S. E. Kim, Y. M. Kim, J. H. Seo, M. C. Shin, M. S. Shin, J. W. Yi, D. H. Shin and H. Kim, J. Med. Food, 2009, 12, 788.

12 S. H. Lim, Afr. J. Pharm. Pharmacol., 2011, 5, 506-514.

13 D. C. Shin, G. C. Kim, S. Y. Song, H. J. Kim, J. C. Yang and B. A. Kim, Korean J. Herbology, 2013, 28, 95-100.

14 X. Wang, J. Liu, N. A. Jin, D. Xu, J. Wang, Y. Han and N. Yin, Exp. Ther. Med., 2015, 9, 1773.

15 C. H. Park, J. S. Noh, J. H. Kim, T. Tanaka, Q. Zhao, K. Matsumoto, N. Shibahara and T. Yokozawa, Biol. Pharm. Bull., 2011, 34, 1559.

16 M. H. Lin, H. K. Liu, W. J. Huang, C. C. Huang, T. H. Wu and F. L. Hsu, J. Agric. Food Chem., 2011, 59, 7743-7751.

17 H. P. Chan, T. Tanaka, H. K. Ji, E. J. Cho, J. C. Park, N. Shibahara and T. Yokozawa, Toxicology, 2011, 290, 14-21.

18 B. Yu, G. Zhang, Y. An and W. Wang, Korean J. Physiol. Pharmacol., 2018, 22, 17.

19 J. Y. Tae, J. S. Chul, H. Jae Sung and K. J. Hee, Chem.-Biol. Interact., 2015, 238, 33-39.

20 Y. M. He, S. Zhu, Y. W. Ge, K. Kazuma, K. Zou, S. Q. Cai and K. Komatsu, J. Nat. Med., 2015, 69, 303-312.

21 D. G. Kang, M. K. Moon and A. S. Lee, Biol. Pharm. Bull., 2007, 30, 1796.

22 W. L. Jiang, X. G. Chen, H. B. Zhu, J. Hou and J. W. Tian, Pharmacology, 2009, 84, 162-170.

23 D. B. Savage, K. F. Petersen and G. I. Shulman, Physiol. Rev., 2007, 87, 507-520.

24 S. E. Kahn, R. L. Hull and K. M. Utzschneider, Nature, 2006, 444, 840.

25 V. T. Samuel and G. I. Shulman, Cell, 2012, 148, 852-871. 26 A. R. Saltiel and J. E. Pessin, Trends Cell Biol., 2002, 12, 65-71. 27 J. A. Engelman, Nat. Rev. Cancer, 2009, 9, 550-562.

28 Y. Min, R. Yan, L. Zhimin, T. Chenchen, J. Yanjun, L. Yerui, Z. Tingting, W. Shaobo, L. Hua and Y. Gangyi, Cell. Signalling, 2015, 27, 2201.

29 G. R. Gandhi, G. Jothi, P. J. Antony, K. Balakrishna, M. G. Paulraj, S. Ignacimuthu, A. Stalin and N. A. Al-Dhabi, Eur. J. Pharmacol., 2014, 745, 201.

30 Z. Zhang, H. Liu and J. Liu, Diabetes Res. Clin. Pract., 2017, S0168-8227(17)30315-7.

31 Z. Chen, C. Wang, Y. Pan, X. Gao and H. Chen, Food \& Function, 2018.

32 X. Su, X. Wang, K. Zhang, S. Yang, Q. Xue, P. Wang and Q. Liu, Gen. Physiol. Biophys., 2014, 33, 295.

33 Y. Chen, Y. Wu, X. Gan, K. Liu, X. Lv, H. Shen, G. Dai and H. Xu, J. Ethnopharmacol., 2016, 194, 850-860.

34 S. Torii, C. Kubota, N. Saito, A. Kawano, N. Hou, M. Kobayashi, R. Torii, M. Hosaka, T. Kitamura and T. Takeuchi, J. Biol. Chem., 2018, 5920-5933. 
35 F. Guerrero-Romero and M. Rodríguez-Moran, Eur. J. Intern. Med., 2014, 25, 265-269.

36 A. M. Melo, R. O. Benatti, L. M. Ignaciosouza, C. Okino, A. S. Torsoni, M. Milanski, L. A. Velloso and M. A. Torsoni, Metab., Clin. Exp., 2014, 63, 682-692.

37 R. Q. Yao, L. Zhang, W. Wang and L. Li, Brain Res. Bull., 2009, 79, 69.

38 H. Q. Xu and H. P. Hao, Biol. Pharm. Bull., 2004, 27, 10141018.

39 H. B. Vaidya, A. A. Ahmed, R. K. Goyal and S. K. Cheema, J. Pharm. Pharm. Sci., 2013, 16, 530-540.

40 K. Du, J. Li, Y. Bai, M. An, X. M. Gao and Y. X. Chang, Food Chem., 2018, 190-196.

41 E. R. Gilbert, Z. Fu and D. Liu, Exp. Diabetes Res., 2011, 2011, 416254.

42 H. J. Kim, K. S. Kim, T. J. Lee and Y. C. Kim, Toxicol. Res., 2009, 25, 93-99.

43 R. Velagaleti, P. Gona, M. Chuang, C. Salton, C. Fox, S. Blease, S. Yeon, W. Manning and C. O'Donnell, Circ. Cardiovasc. Imaging, 2010, 3, 257-263.

44 R. J. Karne, H. Chen and M. J. Quon, Diabetes Care, 2004, 27, 1247.

45 P. D. Loprinzi and K. Abbott, J. Diabetes Metab. Disord., 2014, 13, 47.

46 M. Fizelova, M. Miilunpohja, A. J. Kangas, P. Soininen, J. Kuusisto, M. Alakorpela, M. Laakso and A. Stančáková, Atherosclerosis, 2015, 240, 272-277.
47 C. Guo, C. Zhang, L. Li, Z. Wang, W. Xiao and Z. Yang, Phytomedicine, 2014, 21, 807-814.

48 G. H. Heeba and A. A. Hamza, Life Sci., 2015, 141, 13-19.

49 A. J. Fatani, S. S. Alrejaie, H. M. Abuohashish, A. Alassaf, M. Y. Parmar and M. M. Ahmed, BMC Complementary Altern. Med., 2015, 15(1), 204.

50 A. Repaci, A. Gambineri and R. Pasquali, Mol. Cell. Endocrinol., 2011, 335, 30-41.

51 S. Pillarisetti and U. Saxena, Expert Opin. Ther. Targets, 2004, 8, 401-408.

52 J. Liu, H. C. Yeo, E. Övervikdouki, T. Hagen, S. J. Doniger, D. W. Chu, G. A. Brooks and B. N. Ames, J. Appl. Physiol., 2000, 89, 21.

53 R. Mateos, E. Lecumberri, S. Ramos, L. Goya and L. Bravo, J. Chromatogr. B: Anal. Technol. Biomed. Life Sci., 2005, 827, 7682.

54 A. Song, C. Wang, L. Ren and J. Zhao, Int. J. Mol. Med., 2014, 33, 1671.

55 R. Hemi, Y. Yochananov, E. Barhod, M. Kashermeron, A. Karasik, A. Tirosh and H. Kanety, Diabetes, 2011, 60, 1134.

56 M. Yang, Z. Zhang, C. Wang, K. Li, S. Li, G. Boden, L. Li and G. Yang, Diabetes, 2012, 61, 1959-1968.

57 G. Yu, S. Li, J. Li, Z. Ren, C. Feng and X. Wang, Int. J. Biol. Macromol., 2017, 99, 249.

58 C. Xiao, Q. Wu, J. Zhang, Y. Xie, W. Cai and J. Tan, J. Ethnopharmacol., 2016, 196, 47-57.

59 Y. Gao, M. Zhang, T. Wu, M. Xu, H. Cai and Z. Zhang, J. Agric. Food Chem., 2015, 63, 6019. 